\title{
Collective enhancement of quantum coherence in coupled quantum dot films
}

\author{
Hirokazu Tahara $\odot,{ }^{*}$ Masanori Sakamoto, Toshiharu Teranishi, and Yoshihiko Kanemitsu $\odot^{\dagger}$ \\ Institute for Chemical Research, Kyoto University, Uji, Kyoto 611-0011, Japan
}

(Received 17 February 2021; accepted 12 November 2021; published 17 December 2021)

\begin{abstract}
Colloidal semiconductor quantum dots (QDs) are attractive materials that provide unique photophysics of multiple electron-hole pairs (multiexcitons) in strongly quantum confined systems. Multiexciton phenomena such as efficient Auger recombination have been intensively investigated with respect to individual QDs. However, the cooperative nature of QDs, especially in terms of multiexciton coherence, has not been elucidated thus far. Here, we report the observation of the collective enhancement of quantum coherence in coupled QD films. Using a photocurrent quantum interference technique, we find that the multiexciton quantum coherence in coupled QDs is significantly increased compared to the case of isolated QDs. This cooperative effect is induced by the coherent electronic coupling between QDs. Our results clarify the enhancement mechanism in coupled quantum systems and open the door to advanced optoelectronic applications such as coherent amplifiers and frequency upconverters.
\end{abstract}

DOI: 10.1103/PhysRevB.104.L241405

Owing to continuous efforts for almost four decades, colloidal semiconductor quantum dots (QDs) have grown into extremely excellent nanomaterials. Their photoluminescence quantum efficiencies have increased to nearly $100 \%$ [1-3], and their size-controlled tunability of the band-gap energy has led to significant developments of solution-processed optoelectronic devices, such as light-emitting diodes and solar cells $[4,5]$. These advances have been achieved by understanding intradot multiexciton dynamics, such as efficient Auger recombination [6,7] and carrier multiplication [8-13]. The next challenge is to realize the cooperative effects of a QD ensemble using interdot interactions. In particular, interdot coherence is a new degree of freedom transcending the properties of isolated QDs.

Previous investigations of exciton coherence in QDs have been performed using optical measurements [14-22]. On the other hand, photocurrent measurements are still not widely used to detect coherent dynamics. Since photocurrent signals involve interdot tunneling, photocurrent measurements can be beneficial for clarifying the electronic interactions between QDs. The difficulty in detecting coherent properties by photocurrent measurements originates from the fact that quantum coherence is maintained only during the initial photocarrier generation process, and incoherent carrier scattering governs subsequent photoconductive processes. The most effective solution to this problem is the amplification of the quantum coherence in the initial photocarrier generation process.

\footnotetext{
${ }^{*}$ Corresponding author: tahara.hirokazu.7m@kyoto-u.ac.jp

${ }^{\dagger}$ Corresponding author: kanemitu@ @scl.kyoto-u.ac.jp
}

Published by the American Physical Society under the terms of the Creative Commons Attribution 4.0 International license. Further distribution of this work must maintain attribution to the author(s) and the published article's title, journal citation, and DOI.
Solution-processed QD films provide the most suitable platform for the amplification of quantum coherence in optoelectronic processes, since the electronic wave functions of QDs overlap through direct QD coupling [23-25]. The overlap of electronic wave functions is expected to not only increase the interdot tunneling rate for the photocurrent measurement, but also affect cooperative amplification through the quantum coherence shared by the coupled QDs.

Here, we demonstrate the collective enhancement of quantum coherence in coupled QD films. In order to determine coherent processes from photocurrent signals, we perform photocurrent quantum interference measurements by using a phase stabilization technique of excitation pulses. We clarify that the amplification of multiexciton quantum coherence occurs via coherent electronic coupling between QDs. This cooperative amplification in coupled QDs is quite different from conventional light amplification processes in uncoupled QDs, e.g., amplified spontaneous emission [26,27], superfluorescence [28,29], and lasing [30,31]. Our study provides a unique approach to quantum cooperative amplification by using coherent electronic coupling.

First, we explain the essential idea of the collective enhancement of quantum coherence by increasing the number of quantum states. In order to clarify the role of QD coupling, we focus on a certain condition of coherence called double-quantum coherence, which is generated if QDs absorb two photons. Figure 1(a) shows the generators of doublequantum coherence in three isolated QDs. When the QDs are spatially separated from each other, there are three ways to generate double-quantum coherence by biexcitons. In contrast to the isolated condition, when the QDs are coupled as shown in Fig. 1(b), two excitons can be shared by different QDs. Thus, the number of combinations to generate double-quantum coherence increases to six. The electronic states can be slightly modulated by the QD coupling, but the influence of the resulting energy modulation is 
(a) Isolated QDs

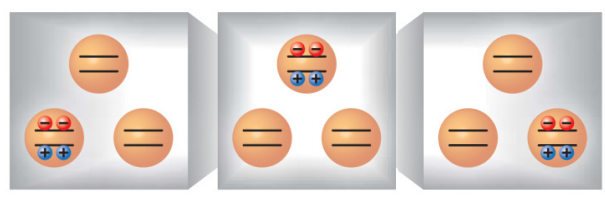

(b) Coupled QDs

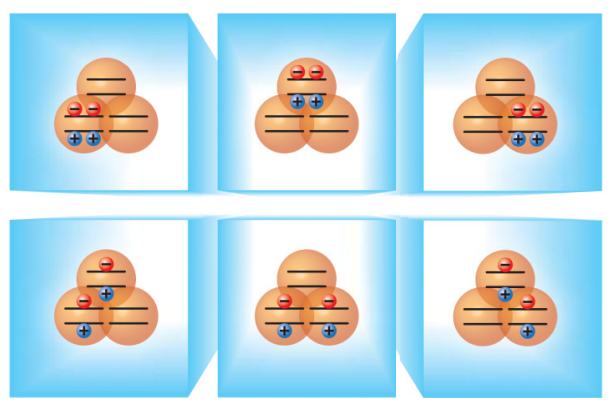

FIG. 1. Generators of double-quantum coherence in (a) isolated and (b) coupled QDs. Single-quantum coherence is generated by a single exciton, i.e., an electron-hole pair, while double-quantum coherence is generated by a biexciton consisting of two electrons and two holes. Isolated QDs have individual quantum states for each QD, while coupled QDs share the same quantum states. Therefore, in coupled QDs, two excitons shared by different QDs, i.e., shared biexcitons, also contribute to double-quantum coherence.

negligibly small at room temperature. Thus, the excitons behave as degenerate states. In terms of the increase in degeneracy, our system behaves similarly to systems where superradiance occurs [28,29,32]. However, the coupling mechanism in our system is different from that of superradiance. Collective photon emission processes such as superradiance can occur even in systems with spatially separated QDs, because of the radiative coupling mechanism. In contrast, the generation of harmonic quantum coherence shared by different QDs requires the overlap between electronic wave functions, i.e., coherent electronic coupling of QDs. Note that collective coherent properties at room temperature are governed by electronic coupling rather than by radiative coupling, since an excitonic polarization usually dephases before radiative coupling occurs. Therefore, the generators of harmonic quantum coherence increase drastically due to the QD coupling, which can be used to enhance quantum coherent signals.

To measure the collective enhancement of quantum coherence in photocurrent signals, we fabricated closely packed QD films using a method described in the literature [33,34] with minor modifications (see Supplemental Material [35]). The device structure is illustrated in Fig. 2(a). Carrier transport layers $(\mathrm{ZnO}$ and $\mathrm{MoOx})$ were fabricated to extract electrons and holes selectively. The surface ligands of $\mathrm{PbS}$ QDs were exchanged from oleic acid to 3-mercaptopropionic acid in order to shorten the distance between the QDs. The total thickness of a stacked QD layer was $130 \mathrm{~nm}$, which was fabricated by multiple cycles of spin-coating. The photocurrent spectrum of the QD film is shown in Fig. 2(b). The peak of the black curve at $1.2 \mathrm{eV}$ corresponds to the ground-state exciton resonance, which exhibits eightfold degeneracy: four equivalent $L$ points with spin degeneracy. We confirmed that (a)

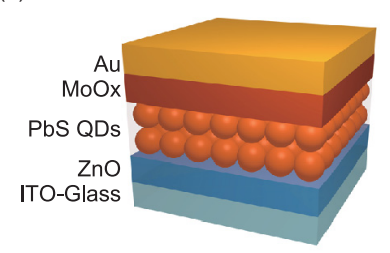

(b)

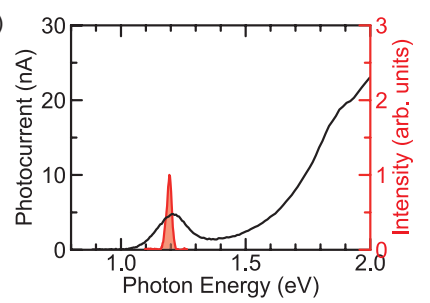

(c)
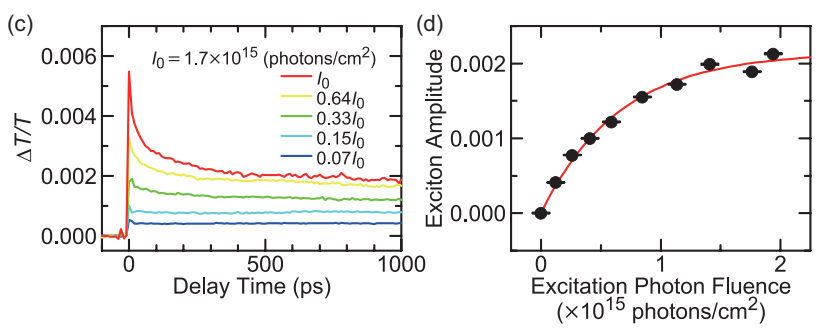

(e)

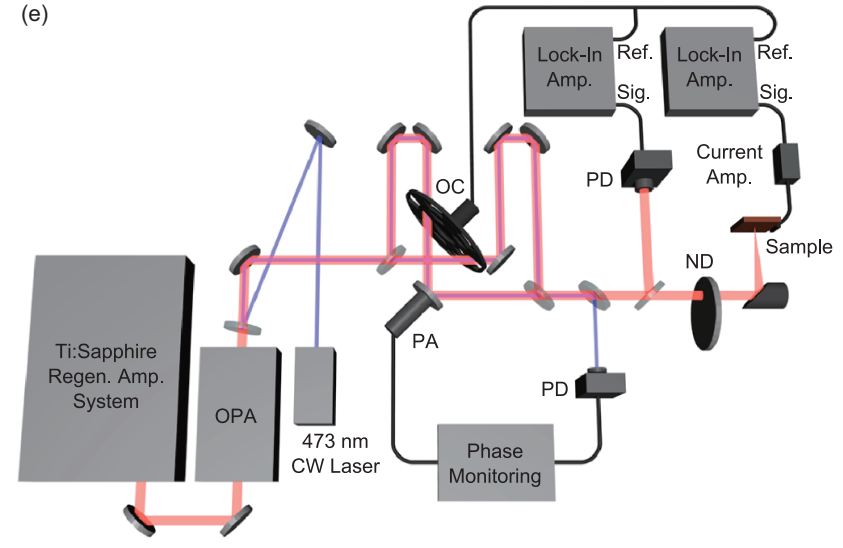

FIG. 2. (a) Illustration of $\mathrm{PbS}$ QD film for photocurrent measurement. (b) Photocurrent spectrum of the QD film (black solid curve). The red shaded area shows the spectrum of the excitation pulse. (c) Transient absorption signals at the exciton resonance for different excitation fluences. (d) Excitation fluence dependence of exciton amplitude in the transient absorption signals. (e) Experimental setup of photocurrent quantum interference measurement with phase-stabilized double pulses. OPA: optical parametric amplifier; OC: optical chopper; PA: piezoelectric actuator; PD: photodetector; ND: neutral density filter.

the difference in the dielectric constant surrounding the QDs does not significantly change the absorption peak energy. We performed the resonant excitation of the ground-state excitons in our experiments. The red shaded area in Fig. 2(b) indicates the spectrum of the excitation pulse.

We determined the QD absorption cross section by using transient absorption measurements. The transient absorption signals in Fig. 2(c) show a fast decay component within the range of 200 ps and a long-lived component whose lifetime is longer than the measurement range. The rapid growth of the fast decay component with increasing excitation photon fluence indicates that the fast decay originates from multiexcitons (see Supplemental Material [35]). In contrast, the long-lived component originates from single excitons. The signal amplitude of the single exciton component exhibits saturation behavior in the excitation photon fluence dependence shown in Fig. 2(d). The QD absorption cross section was determined from a standard analysis of the saturation 

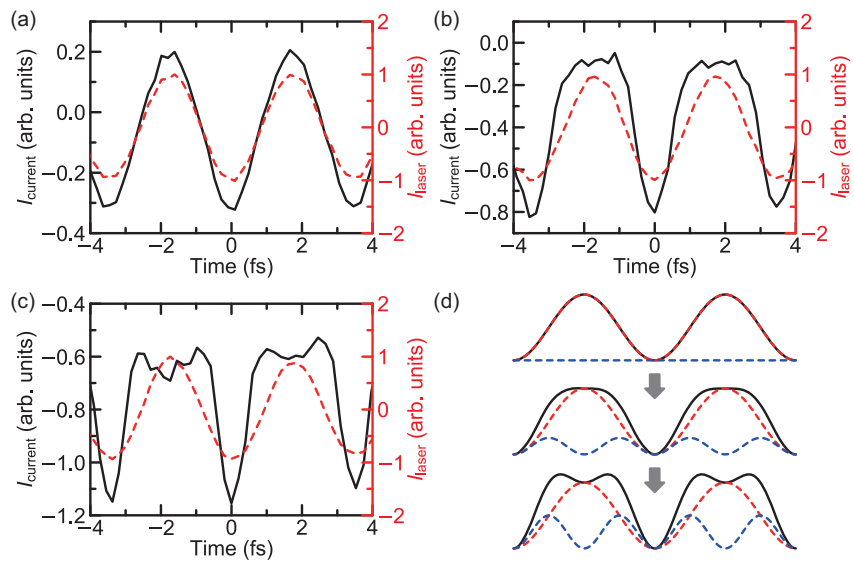

(d)

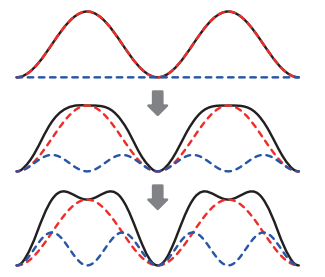

FIG. 3. (a)-(c) Experimental signals of photocurrent and excitation laser pulse interference. The excitation photon fluences are (a) $0.35 \times 10^{15}$, (b) $1.26 \times 10^{15}$, and (c) $2.09 \times 10^{15}$ photons $/ \mathrm{cm}^{2}$. (d) Illustration of structural change in the oscillation. The red (blue) dashed curve describes an oscillation with a fundamental (doubled) frequency. The black solid curve shows their sum. The top of the oscillation splits into a double peak with increasing the amplitude of the oscillation with a doubled frequency.

behavior for single excitons [6,21], which can be described by $1-\exp (-\sigma J)$. Here, $\sigma$ and $J$ denote the QD absorption cross section and the average excitation photon fluence, respectively. The average number of absorbed photons per QD is expressed as $\langle N\rangle=\sigma J$. From the fitting of the saturation behavior shown by the red curve in Fig. 2(d), the cross section is determined to be $1.5 \times 10^{-15} \mathrm{~cm}^{2}$. This value is used in the analysis of the photocurrent signals to determine the average number of excitons per QD.

We performed photocurrent quantum interference measurements using phase-stabilized double pulses to investigate the multiexciton quantum coherence. The experimental setup is shown in Fig. 2(e). The fundamental laser pulse with a photon energy of $\hbar \omega=1.194 \mathrm{eV}$ was generated by an optical parametric amplifier that was pumped by a Ti:sapphire regenerative amplifier. The pulse duration and repetition rate were $100 \mathrm{fs}$ and $1 \mathrm{kHz}$, respectively. To measure photocurrent quantum interference, we prepared a phase-stabilized pulse pair as the excitation pulse pair by using a Mach-Zehnder interferometer [36]. The excitation pulse pair was focused on the sample by a parabolic mirror. The photocurrent signal was amplified by a current amplifier. The photocurrent interference signal was measured by scanning the time interval between the phase-stabilized pulses with the subwavelengthstep actuation of a piezoelectric actuator. At the same time, the optical interference intensity of the excitation laser pulse pair was monitored by a photodetector. Since the measurements are performed at room temperature, multiexciton quantum coherence appears only during the laser pulse irradiation. Therefore, a detection technique in the ultrafast time domain is required to measure multiexciton quantum coherence. We used lock-in amplifiers with a double-chopping technique to detect quantum interference signals during laser pulse irradiation.

The black solid curves in Figs. 3(a)-3(c) show the obtained photocurrent interference signals. The red dashed curves show the interference signals of excitation laser pulses that were measured simultaneously. Under the weak excitation condition shown in Fig. 3(a), the photocurrent oscillation profile corresponds closely to the excitation laser pulse interference. The photocurrent oscillation changes to the flat-top profile shown in Fig. 3(b) with increasing excitation fluence. Under the strong excitation condition, a distinctive feature appears in the oscillation profile. The top of the oscillation splits into a double peak as shown in Fig. 3(c). This double-peak profile is a remarkable feature, since the peak positions deviate from the maximum position of the excitation laser pulse interference. This suggests the emergence of harmonic quantum coherence as shown schematically in Fig. 3(d). Here, for simplicity, double-quantum coherence is described as the lowest-order contribution to the oscillation profile. Since the interference signal due to double-quantum coherence (blue dashed curve) possesses a doubled frequency, its increase changes the oscillation profile. Note that the changes in the oscillation profile cannot be explained by carrier extraction processes. Since carrier extraction processes are much slower than coherent dynamics, they cannot change the ratios between the amplitudes of different harmonic orders. Therefore, an enhancement process changing these ratios is required to explain the emergence of the double-peak profile.

In order to determine the enhancement processes of harmonic quantum coherence, a more detailed analysis including higher-order quantum coherences is required as explained below. We analyzed the photocurrent signals by decomposing them into harmonic quantum interference signals: $A_{n} \cos (n \omega t)$ for $n=$ integer. Here, the frequency $\omega$ corresponds to the dipole oscillation frequency of a single exciton. The amplitude $A_{n}$ describes the contribution of the $n$ th-order harmonic quantum oscillation to the total photocurrent signal. In order to obtain the intrinsic behavior of the $n$ th-order harmonics, the decrease in the carrier extraction efficiency due to Auger recombination and transient photobleaching was taken into account in the analysis (see Supplemental Material [35]). The amplitudes $A_{n}$ are shown by the black solid circles in Fig. 4, where the horizontal axis is the average number of absorbed photons per QD determined from the excitation photon fluence with $\langle N\rangle=\sigma J$. The amplitude of the $\omega$ oscillation, $A_{1}$, shows saturation behavior, while the amplitudes of the $2 \omega$ and higher-order oscillations show accelerating increases that indicate collective enhancement.

The excitation photon fluence dependence can be reproduced by taking into account the generation processes of multiexcitons with the assistance of the QD coupling as explained below. Before discussing the influence of QD coupling, we briefly explain the behavior of harmonic quantum coherence in the absence of the QD coupling. We were not able to detect photocurrent signals from devices without electronic QD coupling, and thus we compare the present results with those of isolated QDs dispersed in toluene. The quantum coherence of such isolated QDs has already been investigated [20,36]. It has been clarified that the amplitudes of harmonic quantum coherence are determined by the probabilities of generating multiexcitons for isolated QDs. By using the standard analysis based on Poisson photon statistics [6,21], the harmonic amplitude $A_{n}$ for isolated QDs is represented as $A_{n}^{\text {(isolated) }}(\langle N\rangle)=A_{\mathrm{s}, n} F_{n}(\langle N\rangle)$ 

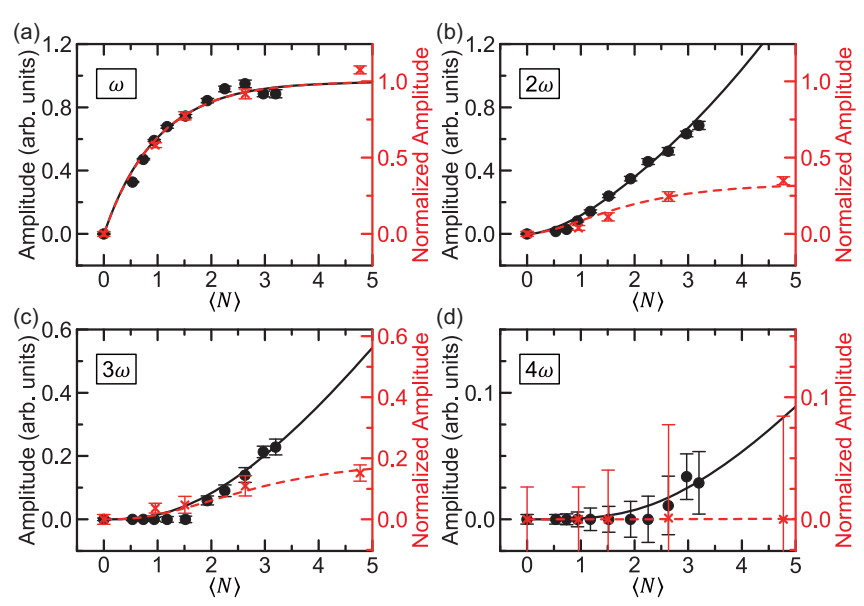

FIG. 4. Amplitudes of harmonic quantum coherence. (a) Single-, (b) double-, (c) triple-, and (d) quadruple-quantum coherences for coupled QDs (black solid circles) and isolated QDs (red crosses) are plotted as a function of the average number of absorbed photons per QD $\langle N\rangle$. The data for isolated QDs are taken from Ref. [20]. Solid (dashed) curves are fits to the experimental data for coupled (isolated) QDs.

with $F_{n}(\langle N\rangle)=\sum_{i \geqslant n} P_{i}(\langle N\rangle)$. It has been shown that the saturated amplitude $A_{\mathrm{s}, n}$ exhibits a power-law decrease with the harmonic order $n$ [20]. The Poisson distribution, $P_{n}(\langle N\rangle)=\langle N\rangle^{n} \exp (-\langle N\rangle) / n$ !, denotes the absorption probability of $n$ photons for an average number of absorbed photons $\langle N\rangle$ per QD. The experimental results for isolated QDs are shown by the red crosses in Fig. 4, which are taken from Ref. [20] and normalized by $A_{\mathrm{s}, 1}$. The calculated results using the above equation (red dashed curves) reproduce the data of the isolated QDs well. In contrast, the experimental results of the coupled QD film for $n \geqslant 2$ obviously deviate from the saturation behavior of isolated QDs. This deviation suggests that the contribution of QD coupling to the harmonic quantum coherence increases with the excitation photon fluence. The key to understanding this feature is the number of excited QDs in contact with each other as shown Fig. 5(a). In the weak excitation regime [left panel in Fig. 5(a)], the excited QDs are sparsely distributed; they behave as isolated QDs. This behavior is not maintained in the strong excitation regime because of the increase in the density of the excited QDs [right panel in Fig. 5(a)]. Thus, the cluster size of the excited QDs becomes larger; that is, the effective number of coupled QDs increases with the excitation fluence.

In order to treat the impact of the QD coupling theoretically, the couplings of the QDs with different oscillation frequencies are taken into account. Here, as the lowest-order contribution to the quantum coherence, we consider the $n \omega$ coherence generated by the coupling of two QDs with $(n-1) \omega$ and $\omega$ oscillations for $n \geqslant 2$. The amplitude of the $n \omega$ oscillation is expressed as

$$
A_{n}^{\text {(coupled) }}(\langle N\rangle)=A_{\mathrm{s}, n}\left\{F_{n}(\langle N\rangle)+g_{\mathrm{c}}\langle N\rangle^{2} F_{n-1}(\langle N\rangle) F_{1}(\langle N\rangle)\right\},
$$

where $g_{\mathrm{c}}$ denotes the QD coupling constant (see Supplemental Material [35]). For $n=1$, the $\omega$ oscillation does not show any coupling enhancement, i.e., $A_{1}^{\text {(coupled) }}(\langle N\rangle)=A_{1}^{\text {(isolated) }}(\langle N\rangle)$,
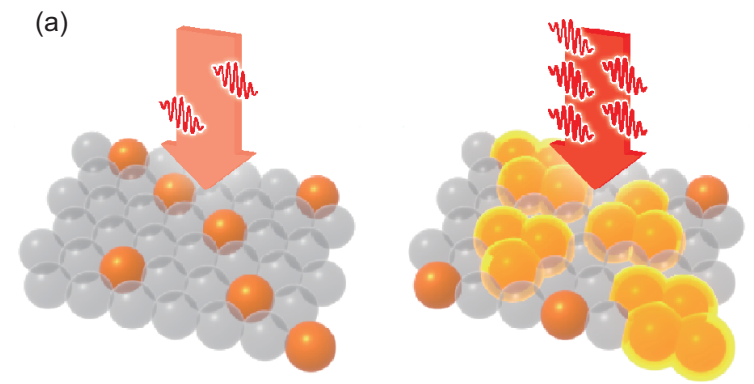

(b)

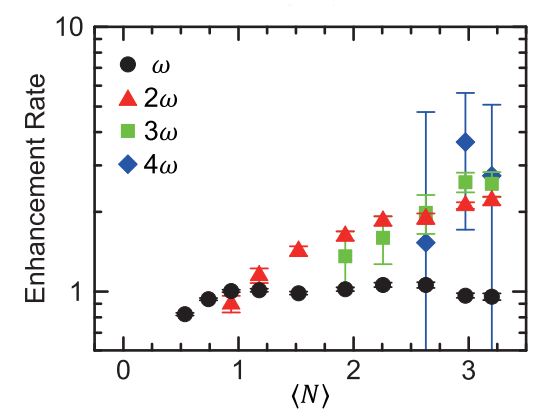

FIG. 5. (a) Illustration of the distribution of excited QDs in a QD layer. The excited QDs are spatially isolated in the weak excitation (left panel), while the excited QDs are in contact with each other in the strong excitation (right panel). Clusters (two or more excited QDs in direct contact) are highlighted in yellow. (b) Enhancement rates of harmonic quantum coherence. The $\omega$ oscillation shows no enhancement that corresponds to the indivisibility of a single frequency, while the $2 \omega$ and higher-order oscillations show enhancements via the QD coupling.

since the single frequency cannot be divided into two or more integer frequencies. The coupling constant $g_{\mathrm{c}}$ describes the effective degree of wave-function overlap between excitons in different QDs, which is determined by the coupling conditions such as the interdot distance, the number of adjacent QDs, and the inhomogeneous size distribution of the QDs. Therefore, the constant $g_{c}$ reflects the actual condition of the QD film including inhomogeneities in the spatial distribution. The calculated results using this equation with $g_{c}=0.11$ are shown by the black solid curves in Fig. 4, which reproduce the accelerating increases of the $2 \omega$ and higher-order oscillations. The obtained saturated amplitudes (see Supplemental Material [35]) show a power-law decrease with the harmonic order $n$, which supports the validity of this analysis. The observed increase of $A_{n}^{\text {(coupled) }}$ for $n \geqslant 2$ in the strong excitation regime shows that the wave-function overlap between excitons in different QDs largely contributes to quantum coherence. The degree of the contribution can be inferred from the difference between the calculated results for the isolated and coupled cases (Fig. 4, dashed and solid curves, respectively).

To clarify the degree of collective enhancement, we determined the enhancement rates by dividing the amplitudes of harmonic quantum coherence by $A_{\mathrm{s}, n} F_{n}(\langle N\rangle)$ as shown in Fig. 5(b). The collective enhancement rates for the $2 \omega$ and higher-order oscillations increase with the excitation photon fluence. This clearly shows that the number of multiexcitons shared by coupled QDs increases with the excitation photon fluence. The enhancement rate of $\sim 3$ was achieved in the strong excitation regime, which allowed us to observe 
quadruple-quantum coherence ( $4 \omega$ oscillation) via quadexcitons.

In conclusion, we demonstrated that the quantum coherence of multiexcitons is enhanced in coupled QD films. The determining factor of the collective enhancement is the quantum state shared by the coupled QDs. We found that the coherent electronic coupling between QDs causes a distinct amplification process of quantum coherence. The observed harmonic quantum coherences can be used as unique energy-upconversion processes, since the harmonic quantum coherences possess higher oscillation frequencies than that of the input infrared light. Furthermore, we clarified that the quantum coherence of multiexcitons is detectable in photocurrent measurements. Thus, our findings open up alternative ways to utilize the coherent electronic coupling in advanced optoelectronic devices.

The authors would like to thank G. Yumoto and F. Sekiguchi for useful discussions. Part of this work was supported by JSPS KAKENHI Grant-in-Aid for Specially Promoted Research (Grant No. JP19H05465) and Early-Career Scientists (Grants No. JP18K13481 and No. JP20K14385).
[1] O. Chen, J. Zhao, V. P. Chauhan, J. Cui, C. Wong, D. K. Harris, H. Wei, H.-S. Han, D. Fukumura, R. K. Jain, and M. G. Bawendi, Nat. Mater. 12, 445 (2013).

[2] B. A. Koscher, J. K. Swabeck, N. D. Bronstein, and A. P. Alivisatos, J. Am. Chem. Soc. 139, 6566 (2017).

[3] D. A. Hanifi, N. D. Bronstein, B. A. Koscher, Z. Nett, J. K. Swabeck, K. Takano, A. M. Schwartzberg, L. Maserati, K. Vandewal, Y. van de Burgt, A. Salleo, and A. P. Alivisatos, Science 363, 1199 (2019).

[4] Y. Shirasaki, G. J. Supran, M. G. Bawendi, and V. Bulović, Nat. Photonics 7, 13 (2013).

[5] J. M. Pietryga, Y.-S. Park, J. Lim, A. F. Fidler, W. K. Bae, S. Brovelli, and V. I. Klimov, Chem. Rev. 116, 10513 (2016).

[6] V. I. Klimov, J. A. McGuire, R. D. Schaller, and V. I. Rupasov, Phys. Rev. B 77, 195324 (2008).

[7] V. I. Klimov, Annu. Rev. Condens. Matter Phys. 5, 285 (2014).

[8] R. D. Schaller and V. I. Klimov, Phys. Rev. Lett. 92, 186601 (2004).

[9] R. J. Ellingson, M. C. Beard, J. C. Johnson, P. Yu, O. I. Micic, A. J. Nozik, A. Shabaev, and Al. L. Efros, Nano Lett. 5, 865 (2005).

[10] A. Shabaev, Al. L. Efros, and A. J. Nozik, Nano Lett. 6, 2856 (2006).

[11] A. Ueda, K. Matsuda, T. Tayagaki, and Y. Kanemitsu, Appl. Phys. Lett. 92, 233105 (2008).

[12] O. E. Semonin, J. M. Luther, S. Choi, H.-Y. Chen, J. Gao, A. J. Nozik, and M. C. Beard, Science 334, 1530 (2011).

[13] A. Shabaev, C. S. Hellberg, and Al. L. Efros, Acc. Chem. Res. 46, 1242 (2013).

[14] P. Borri, W. Langbein, S. Schneider, U. Woggon, R. L. Sellin, D. Ouyang, and D. Bimberg, Phys. Rev. Lett. 87, 157401 (2001).

[15] F. Masia, N. Accanto, W. Langbein, and P. Borri, Phys. Rev. Lett. 108, 087401 (2012).

[16] D. B. Turner, Y. Hassan, and G. D. Scholes, Nano Lett. 12, 880 (2012).

[17] H. Tahara, Y. Ogawa, F. Minami, K. Akahane, and M. Sasaki, Phys. Rev. Lett. 112, 147404 (2014).

[18] H. Utzat, W. Sun, A. E. K. Kaplan, F. Krieg, M. Ginterseder, B. Spokoyny, N. D. Klein, K. E. Shulenberger, C. F. Perkinson, M. V. Kovalenko, and M. G. Bawendi, Science 363, 1068 (2019).

[19] A. Liu, D. B. Almeida, W. K. Bae, L. A. Padilha, and S. T. Cundiff, Phys. Rev. Lett. 123, 057403 (2019).
[20] H. Tahara, M. Sakamoto, T. Teranishi, and Y. Kanemitsu, Phys. Rev. Lett. 119, 247401 (2017).

[21] H. Tahara, M. Sakamoto, T. Teranishi, and Y. Kanemitsu, Nat. Commun. 9, 3179 (2018).

[22] E. Collini, H. Gattuso, Y. Kolodny, L. Bolzonello, A. Volpato, H. T. Fridman, S. Yochelis, M. Mor, J. Dehnel, E. Lifshitz, Y. Paltiel, R. D. Levine, and F. Remacle, J. Phys. Chem. C 124, 16222 (2020).

[23] C. B. Murray, C. R. Kagan, and M. G. Bawendi, Science 270, 1335 (1995).

[24] C. B. Murray, C. R. Kagan, and M. G. Bawendi, Annu. Rev. Mater. Sci. 30, 545 (2000).

[25] C. R. Kagan and C. B. Murray, Nat. Nanotechnol. 10, 1013 (2015).

[26] V. I. Klimov, A. A. Mikhailovsky, S. Xu, A. Malko, J. A. Hollingsworth, C. A. Leatherdale, H.-J. Eisler, and M. G. Bawendi, Science 290, 314 (2000).

[27] K. Wu, Y.-S. Park, J. Lim, and V. I. Klimov, Nat. Nanotechnol. 12, 1140 (2017).

[28] M. Scheibner, T. Schmidt, L. Worschech, A. Forchel, G. Bacher, T. Passow, and D. Hommel, Nat. Phys. 3, 106 (2007).

[29] G. Rainò, M. A. Becker, M. I. Bodnarchuk, R. F. Mahrt, M. V. Kovalenko, and T. Stöferle, Nature (London) 563, 671 (2018).

[30] H.-J. Eisler, V. C. Sundar, M. G. Bawendi, M. Walsh, H. I. Smith, and V. Klimov, Appl. Phys. Lett. 80, 4614 (2002).

[31] O. V. Kozlov, Y.-S. Park, J. Roh, I. Fedin, T. Nakotte, and V. I. Klimov, Science 365, 672 (2019).

[32] R. H. Dicke, Phys. Rev. 93, 99 (1954).

[33] M. A. Hines and G. D. Scholes, Adv. Mater. 15, 1844 (2003).

[34] A. G. Pattantyus-Abraham, I. J. Kramer, A. R. Barkhouse, X. Wang, G. Konstantatos, R. Debnath, L. Levina, I. Raabe, M. K. Nazeeruddin, M. Grätzel, and E. H. Sargent, ACS Nano 4, 3374 (2010).

[35] See Supplemental Material at http://link.aps.org/supplemental/ 10.1103/PhysRevB.104.L241405 for details of the fabrication of coupled QD films, analysis of multiexciton components in transient absorption signals, amplitude of harmonic quantum coherence in photocurrent signals, theoretical model of harmonic quantum coherence in coupled QD systems, and saturated amplitudes of a coupled QD film.

[36] H. Tahara and Y. Kanemitsu, Adv. Quantum Technol. 3, 1900098 (2020). 